\title{
Lung cancer requires multidisciplinary treatment to improve patient survival: A case report
}

\author{
ALDO PEZZUTO $^{1}$, FABRIZIO TERZO ${ }^{2}$, MARIA LAURA GRAZIANI ${ }^{2}$, \\ ALBERTO RICCI $^{1,2}$, PIERDONATO BRUNO ${ }^{1}$ and SALVATORE MARIOTTA ${ }^{1,2}$ \\ ${ }^{1}$ Department of Cardiovascular and Thoracic Disease, Division of Pulmonology, St. Andrew's Hospital; \\ ${ }^{2}$ Department of Clinical and Molecular Medicine, Sapienza University of Rome, I-00189 Rome, Italy
}

Received October 10, 2016; Accepted January 31, 2017

DOI: $10.3892 / \mathrm{ol} .2017 .6511$

\begin{abstract}
The present study reports two cases of lung cancer with the involvement of the pleura. The diagnosis of adenocarcinoma with epidermal growth factor receptor (EGFR) mutation was made following repeated thoracentesis with cytology of pleural fluid and thoracoscopy with pleural biopsies. Talc pleurodesis was successfully performed in both cases subsequent to diagnosis. Following talc pleurodesis, the first patient (62 years old; male; non-smoker) underwent 3 cycles of cisplatin/vinorelbine chemotherapy, with a poor response. Concurrently, due to the presence of an EGFR mutation, treatment with gefitinib was initiated, with the patient achieving a good response for $\sim 12$ months. The residual tumor was treated with stereotactic radiotherapy and the patient continued gefitinib treatment. The patient is presently in good health, has not exhibited any signs of relapse and is continuing gefitinib treatment without side effects. The second patient (53 years old; male ex-smoker) underwent treatment with gefitinib subsequent to talc pleurodesis for a total of 15 months. In addition, radiotherapy (60 Gy) on the residual lesion was performed. Subsequently, second-line therapy with cisplatin/premetrexed was prescribed and followed by maintenance treatment with premetrexed. Three years after diagnosis, the patient did not exhibit any signs of recurrence. These two cases highlight the difficulty in treating advanced stage lung cancer, despite the presence of EGFR mutation. Each lung cancer is different and requires the physician to possess a wide range of knowledge of the therapeutic options available, in addition to careful monitoring in order to adjust the treatment over time. A multidisciplinary approach, involving surgeons, radiation oncologists, pulmonologists and oncologists, is required to optimize the survival and quality of life of patients with lung cancer.
\end{abstract}

Correspondence to: Professor Salvatore Mariotta, Department of Cardiovascular and Thoracic Disease, Division of Pulmonology, St. Andrew's Hospital, Via di Grottarossa 1035-39, I-00189 Rome, Italy

E-mail: salvatore.mariotta@uniroma1.it

Keywords: lung cancer, chemotherapy,radiotherapy, talc pleurodesis, epidermal growth factor receptor mutation, lung cancer survival

\section{Introduction}

Lung cancer is the primary cause of cancer-associated mortality $(1,2)$ and may be classified as non-small cell lung cancer (NSCLC) or small cell lung cancer. NSCLC accounts for $\sim 85 \%$ of all lung cancer cases, of which two-thirds are diagnosed as advanced disease that is not susceptible to therapy and confers a poor prognosis (1). Chemotherapy based on platinum-containing doublets appears to have reached a therapeutic plateau in lung cancer, with median survival times on those receiving this therapy approaching 12 months (2). The development of more effective first-line therapies remains a challenge (2-4).

The results of previous randomized studies suggest that patients with advanced NSCLC with EGFR mutations have a significantly improved progression-free survival (PFS) when treated with EGFR tyrosine kinase inhibitors (TKIs) alone, compared with standard chemotherapy. Conversely, in wild-type EGFR patients, EGFR TKIs treatment was less effective when administered alone compared with chemotherapy (5-11). In patients with lung cancer with metastatic disease of the pleura, pleurodesis with talc has been utilized as an integrated treatment option to inhibit the drainage of pleural fluid and preserve lung function, improving the patient's quality of life (12-15).

Radiotherapy is approved for the treatment of stage IIIA and B lung cancer [as described by the American Joint Committee on Cancer staging system, eighth edition (16)], and for stage IV lung cancer in certain cases (1). Radical radiotherapy for patients with NSCLC can be curative for the minority of patients with early stage disease (17). In addition, when treatment is not curative palliative radiotherapy can be used to aid in pain relief $(17,18)$.

The present study reports two cases of stage IV NCLC, which highlight that a timely and integrated multidisciplinary treatment strategy, including stereotactic radiotherapy and talc pleurodesis, may lead to improved patient survival and quality of life.

\section{Case report}

Patient 1. A male 69-year-old non-smoker was admitted to the Department of Cardiovascular and Thoracic Disease in 
the Division of Pulmonology at St. Andrew's Hospital (Rome, Italy) complaining of exertional dyspnea, a cough and chest pain, in September 2012. The patient had a family history of cancer and the primary comorbidity was arterial hypertension. Following a chest $\mathrm{X}$-ray, high resolution computed tomography (CT) of the thorax was performed (Fig. 1). This revealed a large pleural effusion with pulmonary atelectasis and multiple lymphadenopathies of the mediastinum. The patient subsequently underwent three thoracentesis procedures, with a cytological assessment of adenocarcinoma cells positive for transcription termination factor 1 (TTF1), carcinoembryonic antigen and cytokeratin-7 (CK7), and negative for tumor protein $\mathrm{p} 63$. CT scans demonstrated a re-expansion of the lung and a lesion in the left lower lobe following enhancement with contrast medium injection (Fig. 2A). Bronchoscopy with transbronchial biopsies confirmed the diagnosis of lung adenocarcinoma. Spirometry investigations of lung function identified no significant flow limitation, with forced expiratory volume in $1 \mathrm{sec}$ and forced vital capacity measurements at $>80 \%$ of the predicted level. Hemogasanalysis reported a $\mathrm{pH}$ of 7.38, partial pressure $(\mathrm{Pa})$ of $\mathrm{CO}_{2}$ of $40 \mathrm{mmHg}$ and $\mathrm{PaO}_{2}$ of $80 \mathrm{mmHg}$. A bone scan with radiolabeled technetium highlighted an increased uptake of the compound in the seventh left rib as a metastasis. The patient underwent thoracoscopy and talc pleurodesis (using talc poudrage) in order to maintain integrity of lung function and prevent further thoracentesis. CT confirmed the success of the surgery and the pleural biopsies taken confirmed the metastatic spread of the tumor to the pleura. Chemotherapy was initiated; each cycle was 21 days. Chemotherapy included a cisplatin dose of $70 \mathrm{mg} / \mathrm{m}^{2}$ on days 1 and 21 for 1 cycle, gemcitabine $1,000 \mathrm{mg} / \mathrm{m}^{2}$ on days 1,8 and 21 for 8 cycles, and bevacizumab $7.5 \mathrm{mg} / \mathrm{kg}$ on days 1 and 21 for 4 cycles, resulting in a partial tumor response (Fig. 2B). Additionally, polymerase chain reaction screening of the pleural biopsies identified a deletion of exon 19 of the EGFR gene. Consequently, therapy with gefitinib $(250 \mathrm{mg} /$ day $)$ was initiated, with a good response for 10 months; however, a further positron emission tomography (PET)/CT scan identified a residual lesion through fluorodeoxyglucose $\left({ }^{18} \mathrm{~F}-\mathrm{FDG}\right)$ uptake. Stereotactic radiotherapy with a total dose of 45 Gy divided into 3 fractions was performed on the residual lesion over 3 days. A subsequent PET/CT scan revealed residual inflammation and reduced metabolic activity, with a standardized uptake value $<3$ (Fig. 3). The patient was alive 4 years following diagnosis, exhibited good health and continued gefitinib maintenance with a dose of $250 \mathrm{mg}$ a day, now on the 40th cycle of treatment.

Patient 2. A 52-year-old male ex-smoker with a history of 20 packs/year was admitted to St. Andrew's Hospital in October 2013, complaining of persistent chest pain. The patient's clinical history was negative for comorbidities. The patient received paracetamol without great benefit, while blood counts indicated only mild anemia (hemoglobin, $11.5 \mathrm{~g} / \mathrm{dl}$; normal range, $12-15 \mathrm{~g} / \mathrm{dl}$ ). Following a chest $\mathrm{X}$-ray that revealed a large mass on the left lung, a total body CT scan revealed a mass of $48 \mathrm{~mm}$ in diameter located in the left lower lobe of the lung with mediastinal adenopathies and pleural effusions (Fig. 4A and B). Four thoracentesis procedures, video-assisted thoracoscopy, several biopsies of pleural lesions and talc pleurodesis were performed. A diagnosis of lung adenocarcinoma $\left(\mathrm{CK}^{+}, \mathrm{TTF} 1^{+}\right.$and $\mathrm{p} 63$ negative) with a deletion of exon 19 of the EGFR gene was made, based on the biopsy specimen. Therapy with gefitinib $(250 \mathrm{mg} / \mathrm{day})$ was initiated, continuing for ten months until further disease progression. A total body CT scan 1 year from diagnosis revealed a partial response to therapy, with a reduction in tumor mass of $50 \%$ (Fig. 5A) according to the Response Evaluation Criteria In Solid Tumours version 1.1 (19). However, a ${ }^{18}$ F-FDG-PET scan 3 months later (15 months following diagnosis) identified local progression of the disease (Fig. 5B). Radiotherapy in 20 fractions of 3 Gy each for a total of 60 Gy was performed on the residual lesion over 3 weeks. The patient tolerated the treatment well and continued gefitinib; however, further progression of the disease was observed at 20 months after diagnosis. At this point, cisplatin $\left(60 \mathrm{mg} / \mathrm{m}^{2}, \mathrm{~d} 1\right)$ combined with pemetrexed $\left(500 \mathrm{mg} / \mathrm{m}^{2}\right.$, d1) treatment was initiated for 4 cycles of 21 days. Premetrexed was continued as a maintenance treatment at a dose of $500 \mathrm{mg} / \mathrm{m}^{2}$ (to date, 23 cycles). The patient was alive 3 years after diagnosis and did not complain of respiratory symptoms. The patient's response to therapy was good and thoracic imaging revealed only residual actinic fibrosis, without any sign of disease.

\section{Discussion}

Lung cancer is the second most common cancer in males and the third most common in females; however, it is the primary cause of cancer-associated mortality. In the first three stages of the disease, the various treatment options available (surgery, chemotherapy, radiotherapy and biological therapy) are frequently ineffective depending on the initial stage (1). Stage IV lung cancer confers a poor prognosis, particularly when it co-occurs with pleural effusion (14). In recent years, there have been advancements in techniques that can preserve lung function, such as talc pleurodesis (12). The identification of genetic mutations associated with lung cancer (EGFR, anaplastic lymphoma receptor tyrosine kinase and ROS proto-oncogene 1 receptor tyrosine kinase) and the availability of biological drugs that are less toxic, better tolerated and more effective is improving the prognosis of patients with lung cancer, at least in the short term (5-7). It is a necessity that the therapeutic options available are known by the patients, are the subject of interdisciplinary discussion and are used appropriately.

The cases described in the present report were characterized by a variability in response to different therapeutic options and a relatively good survival time due to the use of all possible treatments. Patient 1 and patient 2 received similar treatment (standard chemotherapy and a biological EGFR-targeted therapy) but exhibited different responses. EGFR-targeted therapy has resulted in a better PFS of patients with NSCLC harboring an activating mutation of EGFR compared with patients with wild-type EGFR (3). The deletion of exon 19 and the L858R mutation of exon 21 are the EGFR alterations that exhibit the best sensitivity to EGFR-TKI treatment $(9,10)$. Patients with NSCLC in the East have been demonstrated to respond better to this treatment compared with those in the West (5). A previous randomized phase III clinical trial investigated the use of the EGFR-TKI gefitinib as a first-line 
A

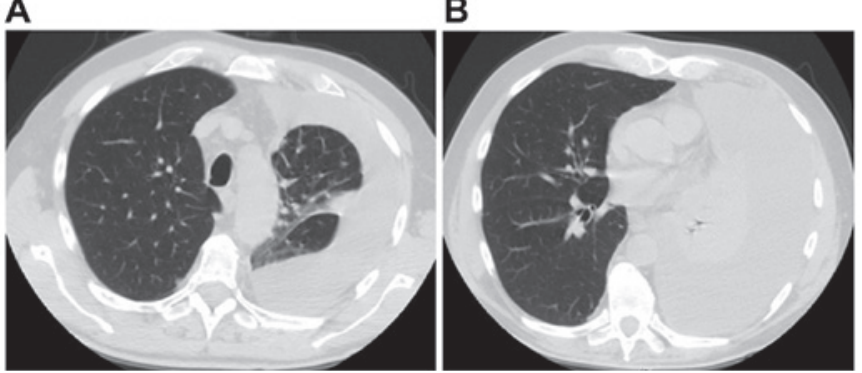

Figure 1. Baseline computed tomography scan of patient 1 revealed (A) a massive pleural effusion on the left lung with (B) sub-atelectasis of the lower lobe

A
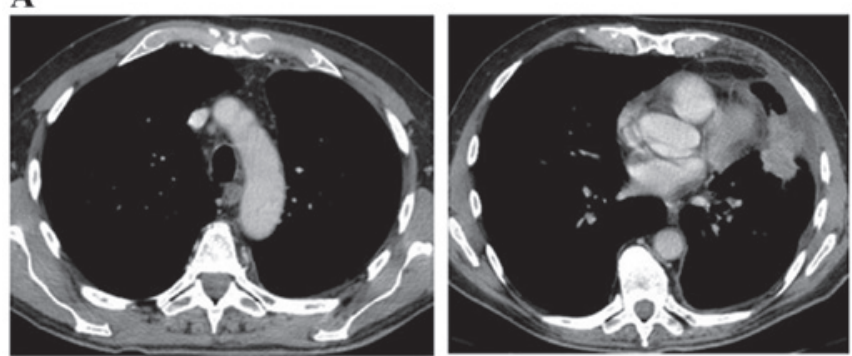

B
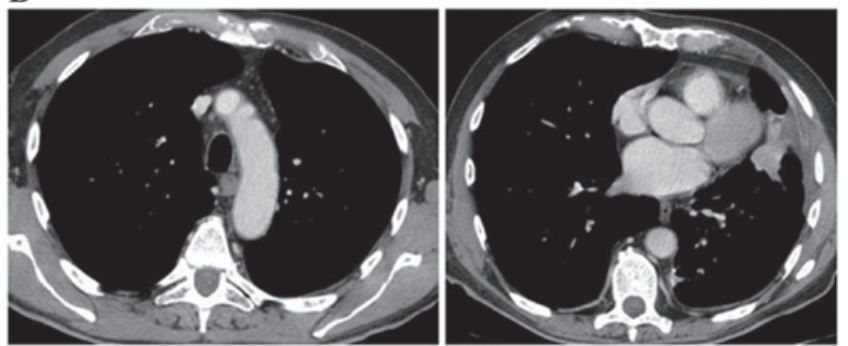

Figure 2. Computed tomography scans of patient 1 (A) following thoracentesis revealed a good re-expansion of the lung and a lesion in the left lower lobe, (B) which was partially reduced subsequent to 4 cycles of chemotherapy.
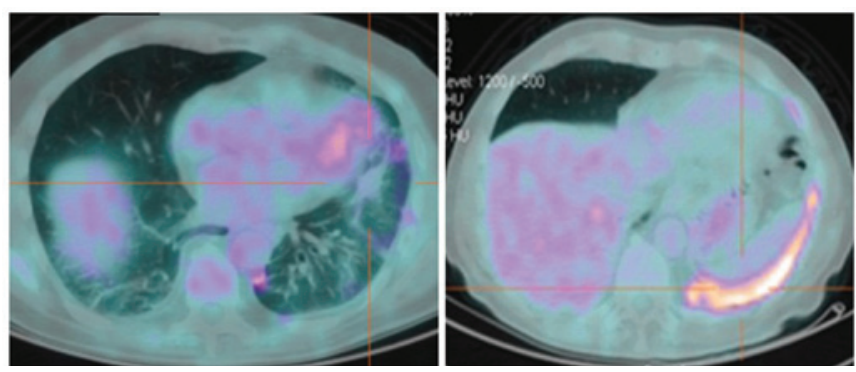

Figure 3. A fuorodeoxyglucose-positron emission tomography-computed tomography scan of patient 1, 6 months after stereotactic radiotherapy, revealed no uptake in the lesion on the lower left lobe of the lung, reduced metabolic activity (standardized uptake value, $<3$ ) and residual inflammation in the pleural space.

treatment for NSCLC compared with standard platinum-based chemotherapy (6), examining PFS and the overall response rate, achieving a median survival time of 16 months. Gefitinib has been demonstrated to be effective in the treatment of lung adenocarcinoma and well-tolerated compared with standard chemotherapy (7). NSCLC cases with the deletion of exon 19
A

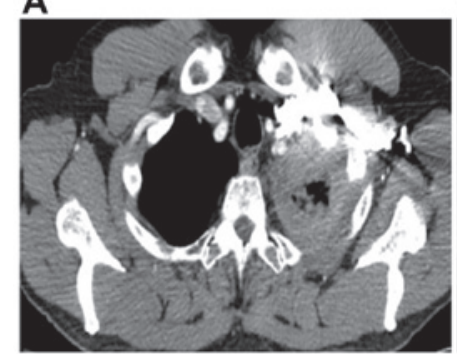

B

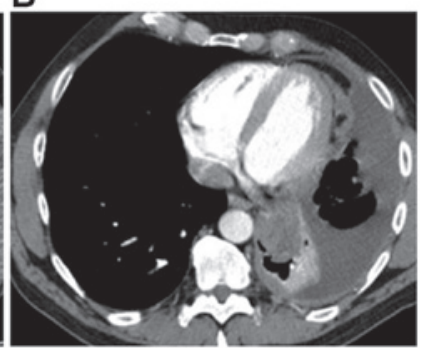

Figure 4. A computed tomography scan of patient 2 revealed (A) a pleural effusion and (B) a lesion in the lower left lobe of the lung.
A
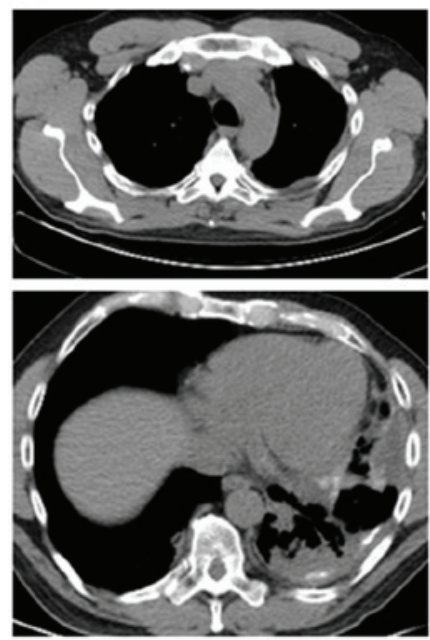

B
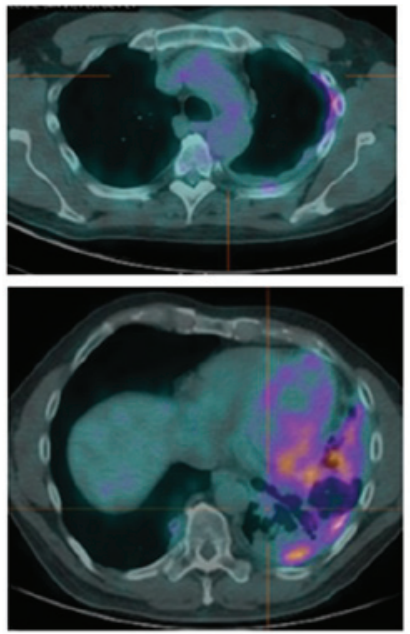

Figure 5. Subsequent to talc pleurodesis and gefitinb treatment for 6 months, patient 2 underwent (A) a CT scan, revealing a pleural thickening, and (B) a ${ }^{18} \mathrm{~F}-\mathrm{FDG}$-positron emission tomography-CT scan, revealing an increased uptake of ${ }^{18} \mathrm{~F}$-FDG in the lesion. CT, computed tomography; ${ }^{18} \mathrm{~F}-\mathrm{FDG}$, fuorodeoxyglucose.

or L858R mutation of exon 21 of EGFR are highly responsive to gefitinib $(7,10)$. Erlotinib is another first generation TKI and it has been demonstrated to be effective in phase III studies as a first-line treatment in patients with EGFR mutation-positive NSCLC (9). In patients with NSCLC with wild-type EGFR, therapy with pemetrexed combined with platinum-base compounds has been identified to improve patient outcomes, such as PFS and overall survival (OS) particularly as a maintenance treatment $(3,4)$. In addition, gefitinib maintenance therapy has been demonstrated to further improve patient survival in a phase III clinical trial $(7,8,10)$.

Clinical guidelines indicate that the use of pemetrexed and bevacizumab should be limited to patients with non-squamous NSCLC histology (20). Bevacizumab can also be used to control brain metastases. The use of maintenance therapy should be considered after 4 cycles of chemotherapy in patients with NSCLC who are responders or do not exhibit disease progression (21). The effect of therapy beyond the third line has not yet been demonstrated. In regards to radiotherapy, guidelines suggest that radiotherapy only be used in stage IV NSCLC for palliative purposes. However, it is a feasible and effective treatment for stage III NSCLC $(1,17)$. 
A previous study investigated the use of sequential or concurrent radio-chemotherapy in unresectable lung cancer, which was successful in improving progression free survival (16). In the cases discussed in the present report, high-dose radiotherapy proved useful in boosting the effects of the EGFR-TKI and to treat residual disease. Radiotherapy could be either palliative or curative, depending on the stage at the time of treatment (18). Radiotherapy in elderly people has been demonstrated to provide some benefit to patients with stage I as a curative treatment and in patients with metastatic stage as palliative treatments to improve the quality of life (17).

The presence of pleural effusion, a common manifestation of NSCLC, worsens the prognosis of patients $(12,13)$. The management of pleural effusions is crucial for improving the patient's quality of life and prognosis (12). Talc pleurodesis is a relatively new method for treating recurrent pleural effusions and is less invasive than other methods $(12,14)$. Pleurodesis can limit the spread of NSCLC and the development of respiratory failure (15). Pleurodesis by thoracoscopy can be performed in certain conditions, including recurrent benign pleural effusion, recurrent pneumothorax and malignant pleural effusion. In the majority of lung cancer cases pleurodesis can slow the progression of the disease (12). The presence of a low glucose level and of low $\mathrm{pH}$ fluid in NSCLC is associated with a poor prognosis $(13,15)$. Zimmer et al (12) compared talc slurry with other chemical substances in a prospective randomized trial, demonstrating that talc was the best agent for the treatment of malignant pleural effusion.

In conclusion, a multidisciplinary approach involving pneumologists, radiotherapists, oncologists and thoracic surgeons, in addition to a variety of treatments, are required for the management of lung cancer. The two patients described in the present report were successfully treated using a range of agents. This suggests that sequential therapies with different agents provide clinical benefit while being well tolerated, improving PFS and OS.

\section{References}

1. Ettinger DS, Wood DE, Akerley W, Bazhenova LA, Borghaei H, Camidge DR, Cheney RT, Chirieac LR, D'Amico TA, Demmy TL, et al: NCCN guidelines insights: Non-Small cell lung cancer, version 4.2016. J Natl Compr Canc Netw 14: 255-264, 2016

2. Molina JR, Yang P, Cassivi SD, Schild SE and Adjei AA: Non-small cell lung cancer: Epidemiology, risk factors, treatment, and survivorship. Mayo Clin Proc 83: 584-594, 2008.

3. Xiao HQ, Tian RH, Zhang ZH, Du KQ and Ni YM: Efficacy of pemetrexed plus platinum doublet chemotherapy as first-line treatment for advanced nonsquamous non-small-cell-lung cancer: A systematic review and meta-analysis. Onco Targets Ther 9: 1471-1476, 2016.

4. Paz-Ares LG, de Marinis F, Dediu M, Thomas M, Pujol JL, Bidoli P, Molinier O, Sahoo TP, Laack E, Reck M, et al: PARAMOUNT: Final overall survival results of the phase III study of maintenance pemetrexed versus placebo immediately after induction treatment with pemetrexed plus cisplatin for advanced nonsquamous non-small-cell lung cancer. J Clin Oncol 31: 2895-2902, 2013.
5. Li G, Gao S, Sheng Z and Li B: The efficacy of single-agent epidermal growth factor receptor tyrosine kinase inhibitor therapy in biologically selected patients with non-small-cell lung cancer: A meta-analysis of 19 randomized controlled trials. Chemotherapy 61: 179-189, 2016.

6. Mitsudomi T, Morita S, Yatabe Y, Negoro S, Okamoto I, Tsurutani J, Seto T, Satouchi M, Tada H, Hirashima T, et al: Gefitinib versus cisplatin plus docetaxel in patients with non-small-cell lung cancer harbouring mutations of the epidermal growth factor receptor (WJTOG3405): An open label, randomised phase 3 trial. Lancet Oncol 11: 121-128, 2010.

7. Maemondo M, Inoue A, Kobayashi K, Sugawara S, Oizumi S, Isobe H, Gemma A, Harada M, Yoshizawa H, Kinoshita I, et al: Gefitinib or chemotherapy for non-small-cell lung cancer with mutated EGFR. N Engl J Med 362: 2380-2388, 2010.

8. Paez JG, Jänne PA, Lee JC, Tracy S, Greulich H, Gabriel S, Herman P, Kaye FJ, Lindeman N, Boggon TJ, et al: EGFR mutations in lung cancer: Correlation with clinical response to gefitinib therapy. Science 304: 1497-1500, 2004.

9. Zhou C, Wu YL, Chen G, Feng J, Liu XQ, Wang C, Zhang S, Wang J, Zhou S, Ren S, et al: Erlotinib versus chemotherapy as first-line treatment for patients with advanced EGFR mutation-positive non-small-cell lung cancer (OPTIMAL, CTONG-0802): A multicentre, open-label, randomised, phase 3 study. Lancet Oncol 12: 735-742, 2011.

10. Mok TS, Wu YL, Thongprasert S, Yang CH, Chu DT, Saijo N, Sunpaweravong P, Han B, Margono B, Ichinose Y, et al: Gefitinib or carboplatin-paclitaxel in pulmonary adenocarcinoma. N Engl J Med 361: 947-957, 2009

11. Yang JC, Sequist LV, Zhou C, Schuler M, Geater SL, Mok T, Hu CP, Yamamoto N, Feng J, O'Byrne K, et al: Effect of dose adjustment on the safety and efficacy of afatinib for EGFR mutation-positive lung adenocarcinoma: Post hoc analyses of the randomized LUX-Lung 3 and 6 trials. Ann Oncol 27: 2103-2110, 2016.

12. Zimmer PW, Hill M, Casey K, Harvey E and Low DE: Prospective randomized trial of talc slurry vs bleomycin in pleurodesis for syntomatic malignant pleural effusions. Chest 112: 430-434, 1997.

13. Cox SE and Katlic MR: Non-intubated video-assisted thoracic surgery as the modality of choice for treatment of recurrent pleural effusions. Ann Transl Med 3: 103, 2015.

14. Rodriguez-Panadero F, Janssen JP and Astoul P: Thoracoscopy: General overview and place in the diagnosis and management of pleural effusion. Eur Resp J 28: 409-422, 2006.

15. Xia H, Wang XJ, Zhou Q, Shi HZ and Tong ZH: Efficacy and safety of talc pleurodesis for malignant pleural effusion: A meta-analysis. PLoS One 9: e87060, 2014.

16. Rami-Porta R, Asamura H, Travis WD and Rusch VW: Lung cancer-major changes in the American Joint Committee on cancer eighth edition cancer staging manual. CA Cancer J Clin 67: 138-155, 2017.

17. Baker S, Dahele M, Lagerwaard F and Senan S: A critical review of recent developments in radiotherapy for non-small cell lung cancer. Radiat Oncol 11: 115, 2016.

18. Dupic G and Bellière-Calandry A: Non-small cell lung cancer irradiation in elderly. Cancer Radiother 20: 322-329, 2016.

19. Eisenhauer EA, Therasse P, Bogaerts J, Schwartz LH, Sargent D, Ford R, Dancey J, Arbuck S, Gwyther S, Mooney M, et al: New response evaluation criteria in solid tumours: Revised RECIST guideline (version 1.1). Eur J Cancer 45: 228-247, 2009.

20. Zappa C and Mousa SA: Non-small cell lung cancer: Current treatment and future advances. Transl Lung Cancer Res 5: 288-300, 2016.

21. Bearz A, Passalacqua R, Alabiso O, Cinieri S, Gridelli C, Cravesana $\mathrm{C}$ and Crinò L: First-line bevacizumab-based therapy in advanced non-squamous non-small-cell lung cancer: Analysis of the Italian patients enrolled in the SAiL study. Clin Drug Investig 32: 755-760, 2012. 